\title{
GATA6-induced FN1 activation promotes the proliferation, invasion and migration of oral squamous cell carcinoma cells
}

\author{
JIANBO ZHAI and GANG LUO \\ Welle Dental, Jingan, Shanghai 200040, P.R. China \\ Received June 8, 2021; Accepted August 12, 2021
}

DOI: $10.3892 / \mathrm{mmr} .2022 .12618$

\begin{abstract}
GATA binding protein 6 (GATA6) is a transcription factor involved in cell fate decision making and tissue morphogenesis and serves a significant role in the progression of a number of types of cancer. The present study aimed to investigate the role and mechanisms underlying the effects of GATA6 in oral squamous cell carcinoma (OSCC). The expression levels of GATA6 were determined in a number of OSCC cell lines and the expression of GATA6 was knocked down to evaluate its role in the proliferation, invasion and migration of OSCC cells. Subsequently, the association between GATA6 and fibronectin 1 (FN1) was investigated using bioinformatics and further verified using dual-luciferase reporter and chromosomal immunoprecipitation assays. Following the overexpression of FN1 in OSCC cells with GATA6 silencing, functional assays were performed to assess the mechanisms underlying GATA6 in OSCC progression. The results of the present study indicated that OSCC cells exhibited markedly upregulated expression levels of GATA6, while knockdown of GATA6 inhibited the proliferation, colony formation, invasion and migration of OSCC cells. In addition, GATA6 regulated FN1 expression levels by binding to the FN1 promoter. The suppressive effects of GATA6 knockdown on the proliferation, colony formation, invasion and migration of OSCC cells were abolished following FN1 overexpression. In conclusion, the findings of the present study demonstrated that GATA6 promoted the malignant development of OSCC cells by binding to the FN1 promotor. These results may contribute to further understanding the pathogenesis of OSCC and provide potential therapeutic targets for the clinical treatment of OSCC.
\end{abstract}

\section{Introduction}

Oral cancer is a major public health concern which accounts for the fourth highest incidence of malignancy (1). Oral squamous cell carcinoma (OSCC) is the most common malignant neoplasm

Correspondence to: Dr Gang Luo, Welle Dental, 5th floor, 1576 West Nanjing Road, Jingan, Shanghai 200040, P.R. China E-mail: luogang009@163.com

Key words: GATA binding protein 6, oral squamous cell carcinoma, fibronectin 1 , invasion, migration of the oral cavity $(2,3)$. A large number of patients with OSCC are often diagnosed at advanced stages and therefore face complex surgical procedures and a poor prognosis (4). Although current research focuses on novel treatment options for OSCC, the prognosis remains poor (5). Thus, investigations into the potential mechanisms underlying the pathogenesis of OSCC may be beneficial for the discovery of novel treatment options for OSCC.

GATA binding protein 6 (GATA6) is a member of the GATA transcription factor family that binds to the $(\mathrm{A} / \mathrm{T})$ $\operatorname{GATA}(\mathrm{A} / \mathrm{G})$ consensus sequence for the activation or inhibition of expression of genes involved in cell fate decision making and tissue morphogenesis (6). GATA6 is an essential factor in modulating cell differentiation and tumor dissemination and is regarded as an independent risk factor for the prognosis of ovarian cancer $(7,8)$. The results of previous studies demonstrate that GATA6 is expressed at high levels in a number of types of cancer, including cholangiocarcinoma, gastric and colorectal cancer (9-11). Additionally, GATA6 is significantly increased in oral carcinoma cell lines (12). The results of a previous study also demonstrated that regulation of microRNA (miRNA/miR)-506 suppresses the expression of GATA6, thus prolonging the development of OSCC (13). However, the specific effects of GATA6 on the proliferation, invasion and migration of OSCC cells remain to be elucidated. Fibronectin 1 (FN1), a glycoprotein present in the extracellular matrix, is closely associated with cellular adhesion and migration (14). The results of a previous study demonstrated that FN1 is considered as a potential biomarker in OSCC associated with the tongue, mouth floor and edentulous ridge (15). Furthermore, inhibition of FN1 suppresses the development of OSCC (16). Thus, the present study aimed to determine whether GATA6 promoted the malignant development of OSCC by inducing FN1 expression.

In the present study, the expression of GATA6 was determined in a number of OSCC cell lines and the subsequent effects on proliferation, invasion and migration of OSCC cells were investigated. Furthermore, the role of FN1 in GATA6 expression and the progression of OSCC was explored.

\section{Materials and methods}

Bioinformatics analyses. The expression of GATA6 and FN1 in OSCC tissues and adjacent tissues was analyzed using the TNMplot database (https://tnmplot.com; the parameters used were Gene-Chip and unpaired samples) (17). The GSE 
dataset GSE146483 (https://www.ncbi.nlm.nih.gov/geo/query/ acc.cgi?acc $=$ GSE146483) consisting of 11 samples (8 OSCC and 3 human normal oral mucosal epithelial keratinocytes) was downloaded from the GEO database (https://www.ncbi. nlm.nih.gov/geo/) and analyzed using GEO2R. Relative gene expression analysis of GATA6 in head and neck squamous cell carcinoma (HNSC) was conducted through LinkedOmics (https://www.linkedomics.org/login.php/) using HiSeq RNA Platform (18). Gene enrichment analysis was conducted by WebGestalt (http://www.webgestalt.org/) using the Gene Set Enrichment Analysis (GSEA) tool and Kyoto Encyclopedia of Genes and Genomes (KEGG) functional database (FDR $<0.25$, adjusted P-value $<0.05)$. The potential transcription factor binding sites in the promoter of FN1 and GATA6 were predicted by JASPAR (http://jaspar.genereg.net/) database.

Cell lines. Human normal oral mucosal epithelial keratinocytes (HOK) and OSCC cell lines (HN4, HN6, SCC-9 and Cal-27), were purchased from the American Type Culture Collection and cultured in DMEM (Gibco; Thermo Fisher Scientific, Inc.) containing $10 \% \mathrm{FBS}$ (Hyclone; Cytiva) at $37^{\circ} \mathrm{C}$ and $5 \% \mathrm{CO}_{2}$.

Cell transfection. Cal-27 cells in the logarithmic phase were inoculated into a 96-well plate and cultured until $70 \%$ confluence was reached. Small interfering (si)RNA targeting GATA6 [si-GATA6-1 (TAGAGTTATTGTGTTAAGAAAGT) and si-GATA6-2 (TAGCGAAGTACTCATAATCTAAT)], the corresponding negative control (si-NC), FN1 overexpression pcDNA3.1 plasmid (Oe-FN1) and the empty vector plasmid (Oe-NC), at a concentration of $\sim 20 \mu \mathrm{M}$, were obtained from Shanghai GenePharma Co., Ltd. Cells were transfected using Lipofectamine $^{\circledR} 3000$ (Invitrogen; Thermo Fisher Scientific, Inc.) according to the manufacturer's protocol. Cells were incubated with $5 \% \mathrm{CO}_{2}$ at $37^{\circ} \mathrm{C}$ and were used in subsequent experiments after $48 \mathrm{~h}$ of transfection. Successful transfection was determined using reverse transcription-quantitative (RT-q) PCR and western blot analysis.

Cell viability assay. A total of $4 \times 10^{4}$ cells/well were seeded into a 96-well plate along with $10 \mu \mathrm{l}$ Cell Counting Kit-8 solution (Shanghai Yeasen Biotechnology Co., Ltd.) and incubated in cell culture medium for $2 \mathrm{~h}$. The viability was subsequently analyzed at a wavelength of $450 \mathrm{~nm}$ in a microplate reader (Promega Corporation).

Colony formation. Cal-27 cells were seeded into 96-well plates at a density of $2 \times 10^{4}$ cells/well and cultured for 10 days for the formation of colonies defined as $>50$ cells/colony. Cells were washed three times with PBS and fixed using $4 \%$ polyoxymethylene for $15 \mathrm{~min}$ at $37^{\circ} \mathrm{C}$. Cells were subsequently stained with crystal violet for $10 \mathrm{~min}$ at $37^{\circ} \mathrm{C}$, images were captured using a light microscope (magnification, x10; Olympus Corporation) and the colonies counted.

Transwell assay. For the evaluation of cell invasion, the upper chamber was pretreated with $100 \mu 1$ Matrigel (BD Biosciences) at $37^{\circ} \mathrm{C}$ for $30 \mathrm{~min}$. A total of $1 \times 10^{5} \mathrm{Cal}-27$ cells suspended in $200 \mu \mathrm{l}$ serum-free DMEM were plated in the upper chamber of a 24-well $8-\mu \mathrm{m}$ pore Transwell insert (Costar; Corning, Inc.). Subsequently, DMEM containing 10\% FBS was placed in the basolateral chamber. Invaded cells were fixed with $4 \%$ paraformaldehyde for $20 \mathrm{~min}$ at room temperature, washed three times with PBS and stained with crystal violet for $10 \mathrm{~min}$ at room temperature. The cells were observed under a light microscope (Olympus Corporation) (magnification, x100) for statistical analysis.

Wound healing assay. Cal-27 cells were inoculated into 96-well plates and incubated until $80 \%$ confluence was reached. A $10-\mu 1$ pipette tip was used to create a wound on the surface of the Cal-27 cells. Cells were subsequently cultured in serum-free DMEM. Following an indicated time after the scratch, the migratory ability of Cal-27 cells was observed using a light microscope (Olympus Corporation).

Dual-luciferase reporter assay. Wild-type (WT) or mutant (MUT) promoter site of FN1 was introduced into the pGL3-Basic vector (Promega Corporation) to make WT-FN1 promoter and MUT-FN1 promoter plasmids. The luciferase reporter plasmids and GATA6-expressing plasmid or empty vector were co-transfected into Cal-27 cells using Lipofectamine ${ }^{\circledR} 3000$ (Invitrogen; Thermo Fisher Scientific, Inc.), according to the manufacturer's protocol. Luciferase activities were measured $48 \mathrm{~h}$ after transfection using the Dual-Luciferase Reporter Assay kit (Promega Corporation). The relative luciferase activity was normalized to Renilla luciferase activity.

Chromatin immunoprecipitation (CHIP) assay. The binding of GATA6 to the FN1 promoter was examined using a CHIP assay kit (Beyotime Institute of Biotechnology), according to the manufacturer's protocol. Cells were cross-linked using $1 \%$ formaldehyde for $15 \mathrm{~min}$ at room temperature and cell lysates in SDS lysis buffer (GBCBIO Technologies, Inc.) were sonicated (10 sec, five times) on ice to achieve 200-1,000 bp chromatin fragments and centrifuged at $4^{\circ} \mathrm{C}, 16,100 \mathrm{x}$ g for 10 min. CHIP was conducted following incubation with the anti-GATA6 antibody (cat. no. 5851T; 1:50; Cell Signaling Technology, Inc.). The recuperated DNA fragments were evaluated via qPCR. The primer sequences were: FN1, forward 5'-CCGAAGAGAGGTGACGCAAT-3' and reverse 5'-GAGTGGCTGGACTTGTGTGA-3'.

$R T-q P C R$. Total RNA was extracted from $1 \times 10^{4}$ cells using TRIzol $^{\circledR}$ reagent (Thermo Fisher Scientific, Inc.) according to the manufacturer's protocol. Total RNA was reverse transcribed into cDNA using a reverse transcriptase cDNA synthesis kit (PrimeScript RT Reagent kit; Takara Bio, Inc.) according to the manufacturer's protocol. The temperature protocol was as follows: $70^{\circ} \mathrm{C}$ for $5 \mathrm{~min}, 37^{\circ} \mathrm{C}$ for $5 \mathrm{~min}$ and $42^{\circ} \mathrm{C}$ for $1 \mathrm{~h}$. qPCR was subsequently performed using a SYBR-Green PCR kit (Takara Bio, Inc.) on an ABI 7500 Real-Time PCR detection instrument (Thermo Fisher Scientific, Inc.) according to the manufacturer's protocol. The following thermocycling conditions were used: pre-denaturation at $95^{\circ} \mathrm{C}$ for $10 \mathrm{~min}$, denaturation at $95^{\circ} \mathrm{C}$ for $15 \mathrm{sec}$, annealing at $60^{\circ} \mathrm{C}$ for $1 \mathrm{~min}$ (40 cycles) and extension at $72^{\circ} \mathrm{C}$ for $1 \mathrm{~min}$. The primer sequences for PCR were: GATA6 5'-TGCAATGCTTGTGGAC TCTA-3' (forward) and 5'-GTGGGGGAAGTATTTTTGCT-3' (reverse); FN1 5'-CGGTGGCTGTCAGTCAAAG-3' (forward) and 5'-AAACCTCGGCTTCCTCCATAA-3' (reverse); GAPDH 5'-GGCTCATGACCACAGTCCATG-3' (forward) 
A
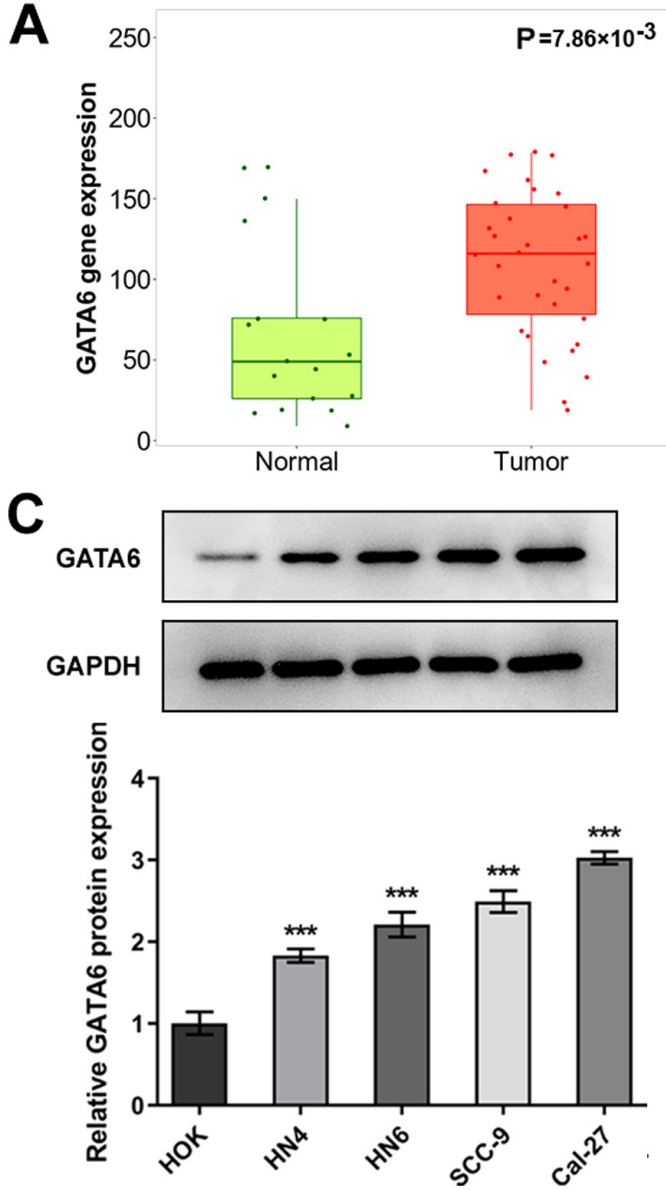

B

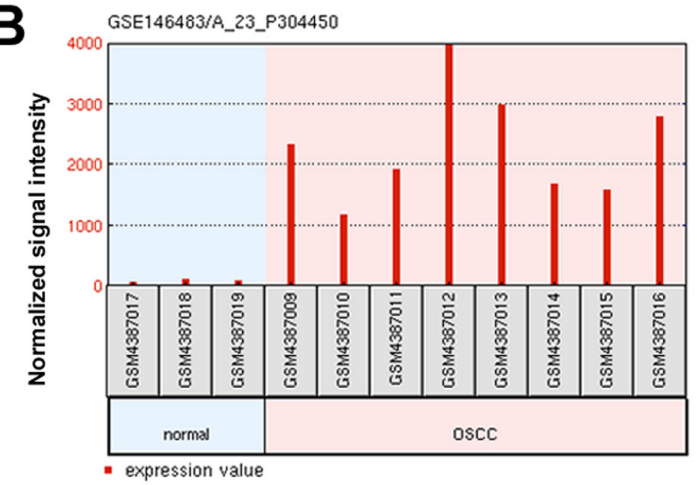

D

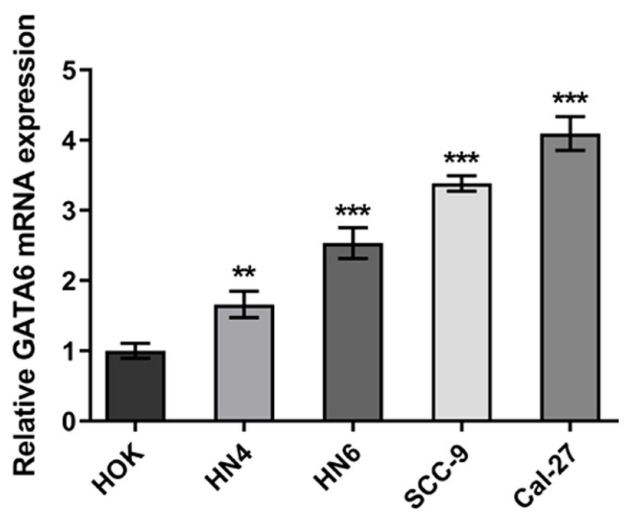

Figure 1. GATA6 is highly expressed in OSCC tissues and cells. (A) TNMplot data was employed to analyze the expression of GATA6 in OSCC tissues and adjacent tissues. (B) The GSE dataset GSE146483 (https:/www.ncbi.nlm.nih.gov/geo/query/acc.cgi?acc=GSE146483) consisting of 11 samples (8 OSCC and 3 human normal oral mucosal epithelial keratinocytes) was downloaded from the GEO database and analyzed using GEO2R. GATA6 expression was detected in HOK and OSCC cell lines using (C) western blot analysis and (D) reverse transcription-quantitative PCR. ${ }^{* *} \mathrm{P}<0.01,{ }^{* * *} \mathrm{P}<0.001$ vs. HOK. GATA6, GATA binding protein 6; OSCC, oral squamous cell carcinoma.

and 5'-TCAGCTCTGGGATGACCTTG-3' (reverse). mRNA levels were quantified using the $2^{-\Delta \Delta C q}$ method and normalized to the internal reference gene, GAPDH (19). All experiments were performed in triplicate.

Western blot analysis. Total proteins were obtained from Cal-27 cells $\left(1 \times 10^{4}\right)$ using RIPA lysis buffer (Beyotime Institute of Biotechnology). Total protein was quantified using a BCA kit (Beyotime Institute of Biotechnology) and $40 \mu \mathrm{g}$ protein sample was separated by SDS-PAGE on a $10 \%$ gel. The separated proteins were subsequently transferred onto PVDF membranes and blocked for $1 \mathrm{~h}$ at room temperature with $5 \%$ skimmed milk. The membranes were incubated overnight at $4^{\circ} \mathrm{C}$ with the following primary antibodies: Anti-GATA6 (cat. no. 5851T; 1:1,000; Cell Signaling Technology, Inc.), anti-FN1 (cat. no. 26836S; 1:1,000; Cell Signaling Technology, Inc.), anti-Ki67 (cat. no. ab16667; 1:1,000; Abcam), anti-proliferating cell nuclear antigen (PCNA; 13110T; 1:1,000; Cell Signaling Technology, Inc.), anti-matrix metalloproteinase-2 (MMP2; cat. no. 40994S; 1:1,000; Cell Signaling Technology, Inc.), anti-MMP9 (cat. no. 13667T; 1:1,000; Cell Signaling Technology, Inc.) and anti-GAPDH (cat. no. 5174T; 1:1,000; Cell Signaling Technology, Inc.). Following the primary antibody incubation, the membranes were incubated with horseradish peroxidase-conjugated secondary antibody (cat. no. 7074S; 1:3,000; Cell Signaling Technology, Inc.) for $1 \mathrm{~h}$ at room temperature and washed three times with PBS. Proteins bands were visualized using enhanced chemiluminescence (Thermo Fisher Scientific, Inc.). The relative intensity of each band was semi-quantified using ImageJ software (version 1.52r; National Institutes of Health). GAPDH was used as the loading control.

Statistical analysis. Data were presented as the mean \pm standard deviation of three independentexperiments simultaneously. Results were analyzed using GraphPad Prism software (version 8.0; GraphPad Software, Inc.). Statistical differences were evaluated using an unpaired Student's t-test or one-way ANOVA followed by a Tukey's post-hoc test. Pearson's correlation analysis was utilized to confirm the correlation between GATA6 and FN1. P<0.05 was considered to indicate a statistically significant difference.

\section{Results}

GATA6 is markedly upregulated in OSCC tissues and cells. To investigate the role of GATA6 in OSCC development, TNMplot data was employed to analyze the expression of GATA6 in OSCC tissues and adjacent tissues. As shown in Fig. 1A, 
A

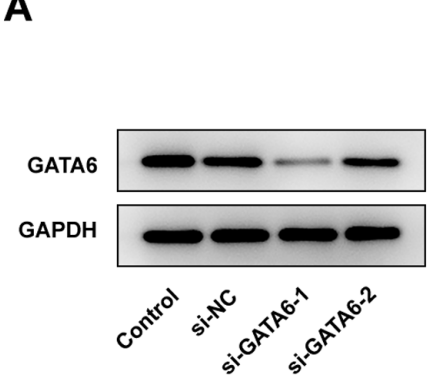

C

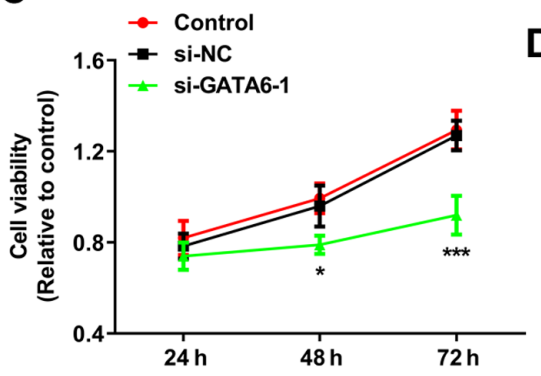

$\mathbf{F}$

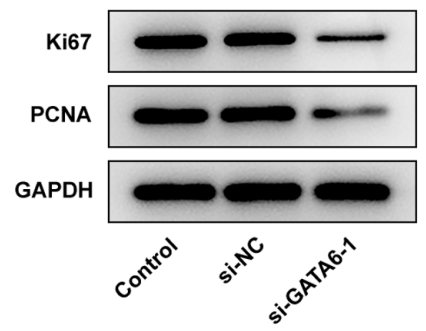

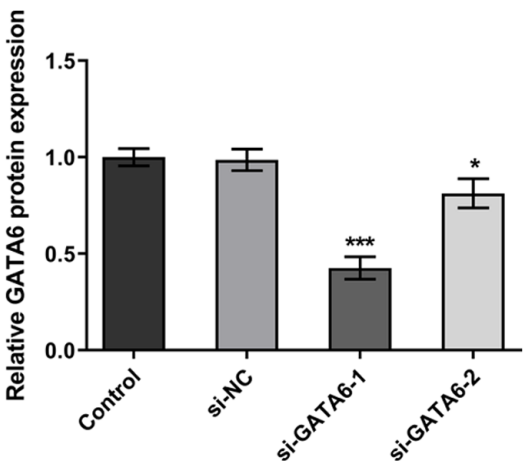

D

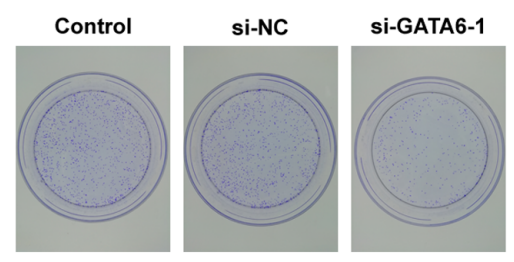

E
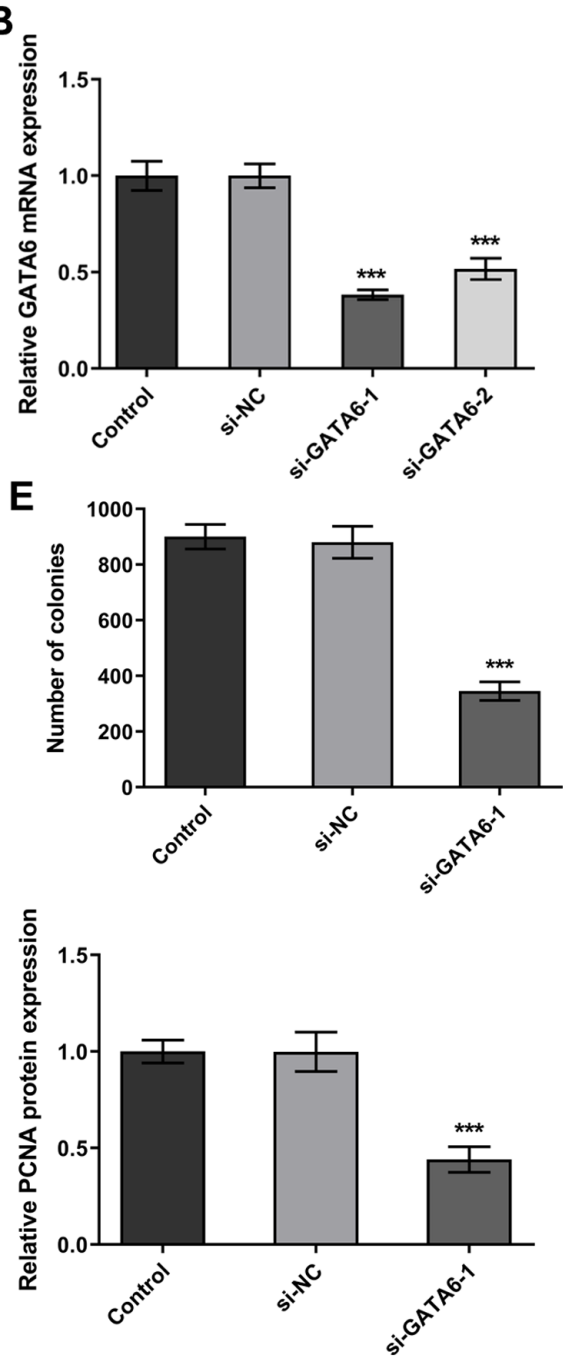

Figure 2. GATA6 silencing inhibits the proliferation and colony formation of oral squamous cell carcinoma cells. (A and B) GATA6 expression levels were analyzed in Cal-27 cells using (A) western blot analysis and (B) reverse transcription-quantitative PCR following transfection with si-GATA6. (C) Cell viability was investigated using a Cell Counting Kit-8 assay. (D and E) Cell proliferation was investigated using a colony formation assay. (F) Western blot analysis was used to assess the expression levels of proliferation-related proteins Ki67 and PCNA. ${ }^{*} \mathrm{P}<0.05,{ }^{* * * *} \mathrm{P}<0.001$ vs. si-NC. GATA6, GATA binding protein 6; si, small interfering RNA; NC, negative control; PCNA, proliferating cell nuclear antigen.

GATA6 was highly expressed in tumor group as comparison to the normal group. The expression levels of GATA6 in eight OSCC cells and three human normal oral mucosal epithelial keratinocytes and were determined using the GEO2R analysis in an independent cohort (GSE146483). As demonstrated in Fig. 1B, GATA6 expression was markedly upregulated in OSCC group compared with the human normal oral mucosal epithelial keratinocytes (normal) group. Subsequently, RT-qPCR and western blot analyses were used to assess the expression levels of GATA6 in OSCC and HOK cell lines. As demonstrated in Fig. 1C and D, the expression levels of GATA6 protein and mRNA were high in OSCC cells compared with the HOK group. The highest expression levels of GATA6 were observed in Cal-27 cells, which were therefore used for subsequent analyses. These results indicated that GATA6 was upregulated in OSCC tissues and cells.

GATA6 silencing inhibits the proliferation, invasion and migration of OSCC cells. Changes in cellular function were examined in Cal-27 cells following transfection with siRNA for the knockdown of GATA6. si-GATA6-1 was selected for subsequent transfections due to a high knockdown efficiency (Fig. 2A and B). Notably, Cal-27 cells transfected with si-GATA6-1 demonstrated a suppressed ability to proliferate and form colonies and downregulation of proliferation-related proteins, Ki67 and PCNA, compared with the si-NC group (Fig. 2C-F). Furthermore, the levels of invasion and migration were reduced in Cal-27 cells transfected with si-GATA6-1 and the expression levels of MMP2 and MMP9 were also reduced, compared with the si-NC group (Fig. 3). Collectively, the results of the present study suggested that GATA6 silencing inhibited the proliferation, invasion and migration of OSCC cells.

GATA6 transcriptionally regulates FN1 levels by binding to the FN1 promoter. To examine the transcriptional role of GATA6 in OSCC, the LinkedOmics database was used to predict genes associated with GATA6. A squamous cell carcinoma of the head and neck dataset was used as a substitute as no OSCC dataset currently exists. Genes positively associated with GATA6 were consistent with those negatively associated 
A
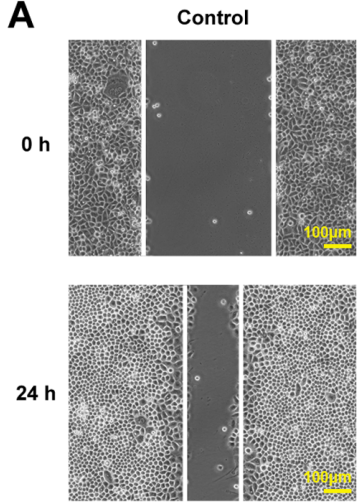

C

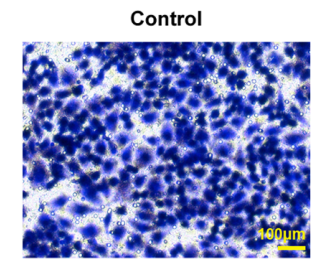

E

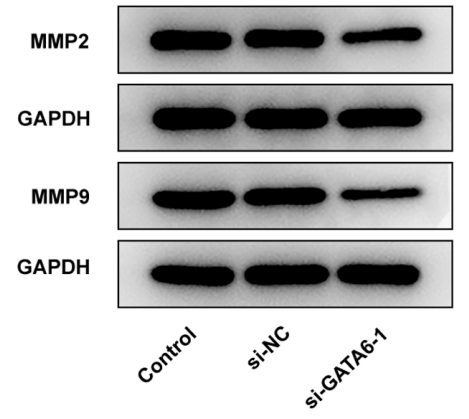

si-NC
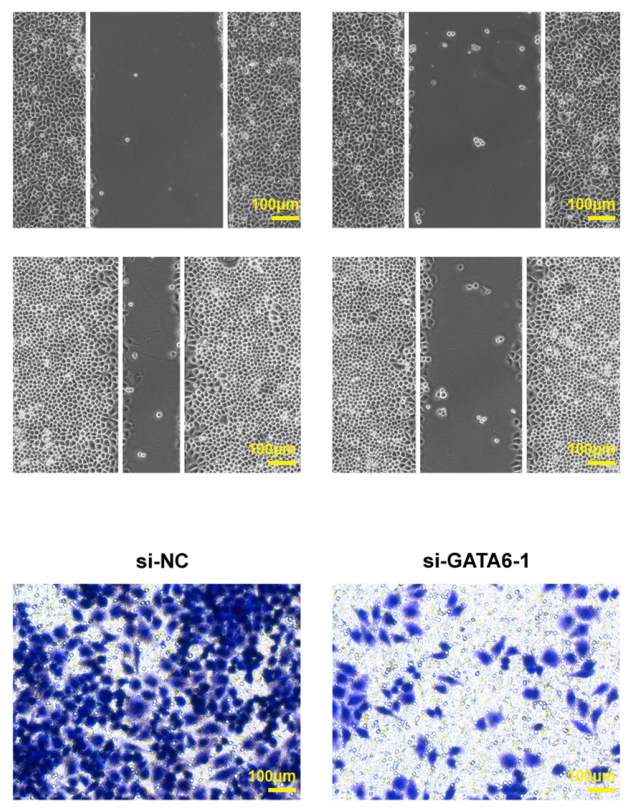

si-GATA6-1
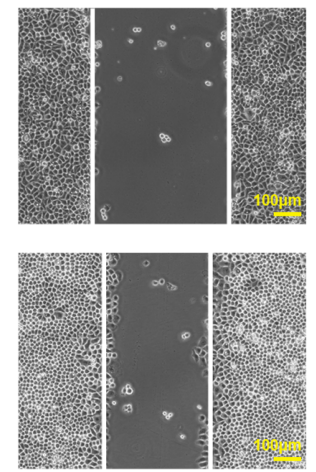

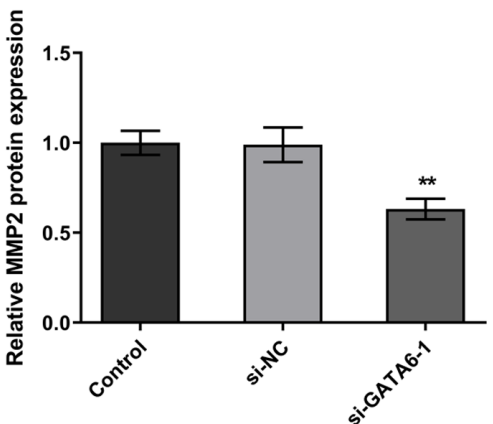

B
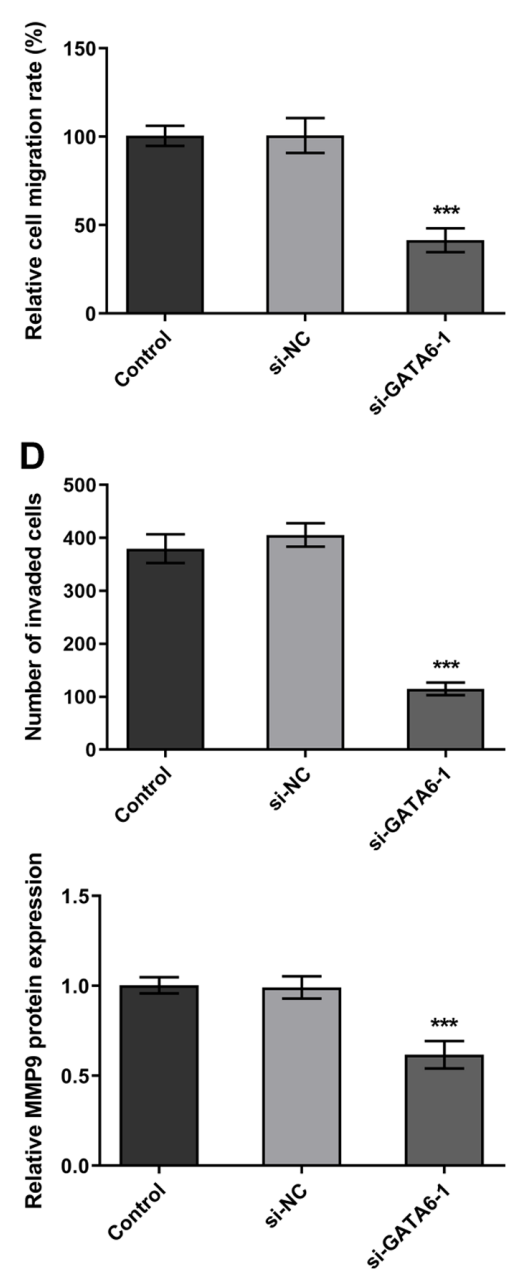

Figure 3. GATA6 silencing suppresses the invasion and migration of oral squamous cell carcinoma cells. (A and B) Cell migration and (C and D) invasion were evaluated using wound healing and Transwell assays, respectively. Magnification, x100. (E) MMP2 and MMP9 expression levels were measured using western blot analysis. ${ }^{* *} \mathrm{P}<0.01,{ }^{* * *} \mathrm{P}<0.001$ vs. si-NC. GATA6, GATA binding protein 6; si, small interfering RNA; NC, negative control.

with GATA6 (Fig. 4A). Furthermore, gene set enrichment analysis (GSEA) revealed that GATA6 was positively associated with extracellular matrix (ECM)-receptors and the closest association was observed between FN1 and GATA6 (Fig. 4B). Subsequently, the expression of FN1 in OSCC tissues and adjacent tissues was evaluated with TNMplot data. It was found that FN1 level was markedly elevated in the tumor group when compared to the normal group (Fig. 5A). The binding site of GATA6 on the promoter region of FN1 was presented in Fig. 5B. Furthermore, dual-luciferase reporter plasmids were activated following GATA6 overexpression (Fig. 5C). CHIP assays performed using Cal-27 cell extracts demonstrated a notable enrichment of the FN1 promoter sequence through immunoprecipitation with an anti-GATA6 antibody, compared with the control IgG antibody (Fig. 5D). Additionally, western blotting and RT-qPCR revealed that the expression levels of FN1 were markedly decreased following GATA6 silencing (Fig. 5E and F). Collectively, these results demonstrated that GATA6 transcriptionally activates FN1 expression by binding to the FN1 promoter.

GATA6 silencing inhibits the proliferation, invasion and migration of OSCC cells by regulating FN1 expression. To further understand the mechanism underlying GATA6 in the progression of OSCC, FN1 was overexpressed in Cal-27 cells. Transfection efficiency was indicated using western blotting and RT-qPCR (Fig. 6A and B). Cal-27 cells transfected with both si-GATA6-1 and Oe-FN1 exhibited high levels of cell viability, proliferation and colony formation and high expression levels of Ki67 and PCNA, compared with Cal-27 cells transfected with si-GATA6-1 + Oe-NC. These results highlighted that Oe-FN1 reversed the effects of si-GATA6-1 on the proliferation of Cal-27 cells (Fig. 6C-F). Additionally, transfection with Oe-FN1 abolished the suppressive effects of si-GATA6-1 on the migration and invasion of Cal-27 cells relative to the si-GATA6-1 + Oe-NC group (Fig. 7). The results of the present study demonstrated that GATA6 silencing suppressed the proliferation, invasion and migration of OSCC cells by regulating FN1.

\section{Discussion}

GATA6 serves an essential role in enhancing and suppressing the development of tumors (20). The differentiation and self-renewal of lung and colon cancer stem cells is promoted 

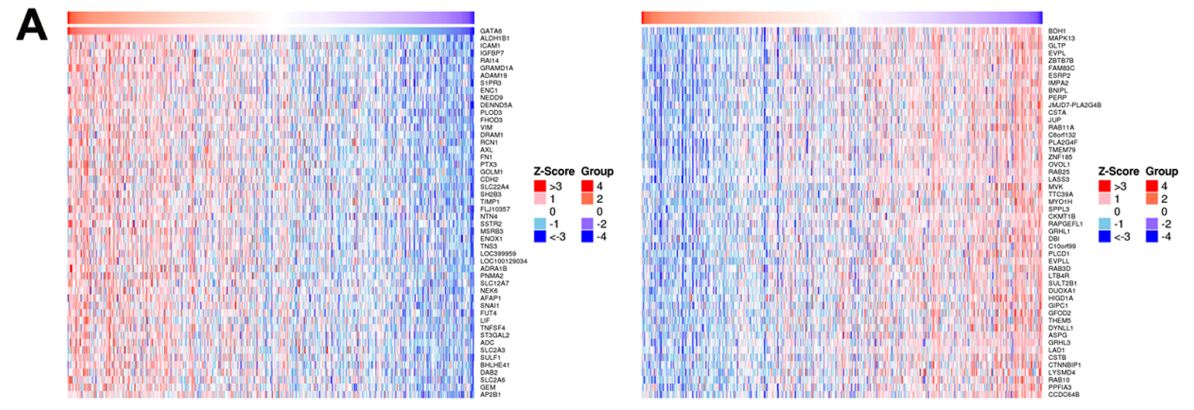

B
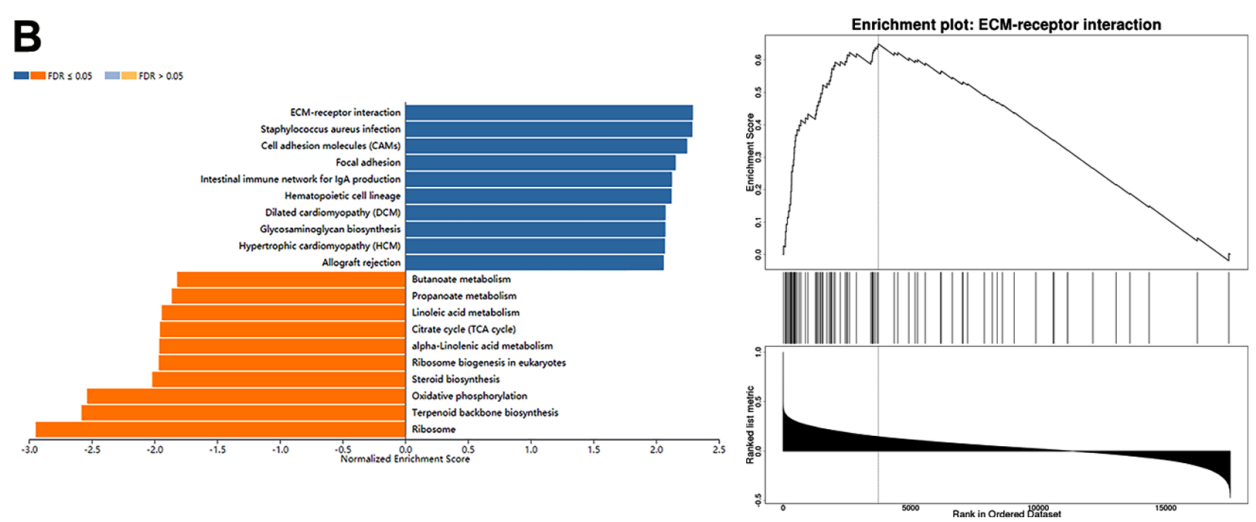
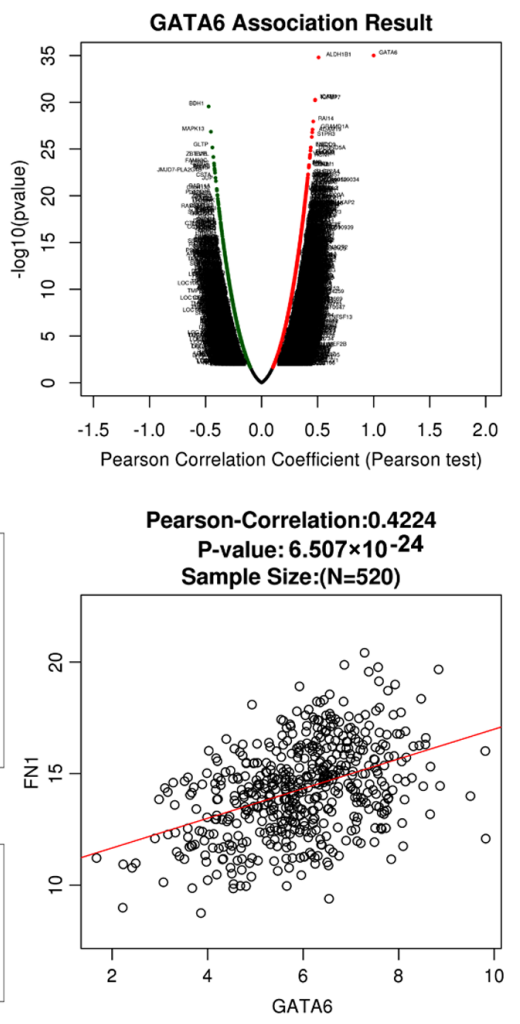

Figure 4. Bioinformatics analyses of GATA6-associated genes. (A) Relative gene expression analysis of GATA6 in head and neck squamous cell carcinoma was conducted through LinkedOmics (https://www.linkedomics.org/login.php/) using HiSeq RNA Platform (17). (B) Gene enrichment analysis was conducted by WebGestalt (http://www.webgestalt.org/) using Gene Set Enrichment Analysis tool and Kyoto Encyclopedia of Genes and Genomes functional database (FDR <0.25, adjusted P-value <0.05). And association between GATA6 and ECM-receptors revealed the highest association between FN1 and GATA6. GATA6, GATA binding protein 6; ECM, extracellular matrix; FN1, fibronectin 1.

B

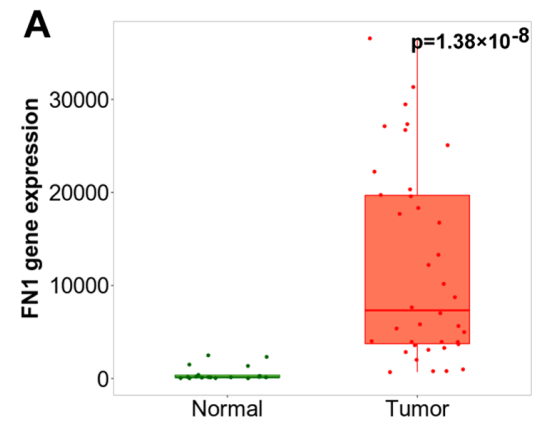

C

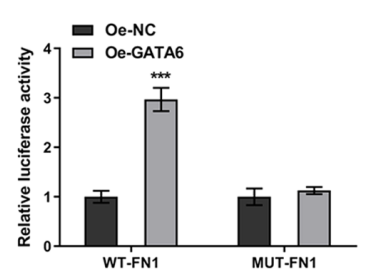

D

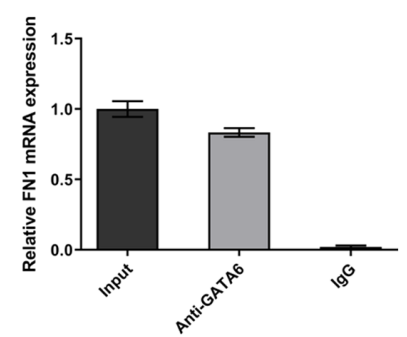

Canonical GATA6 binding motif
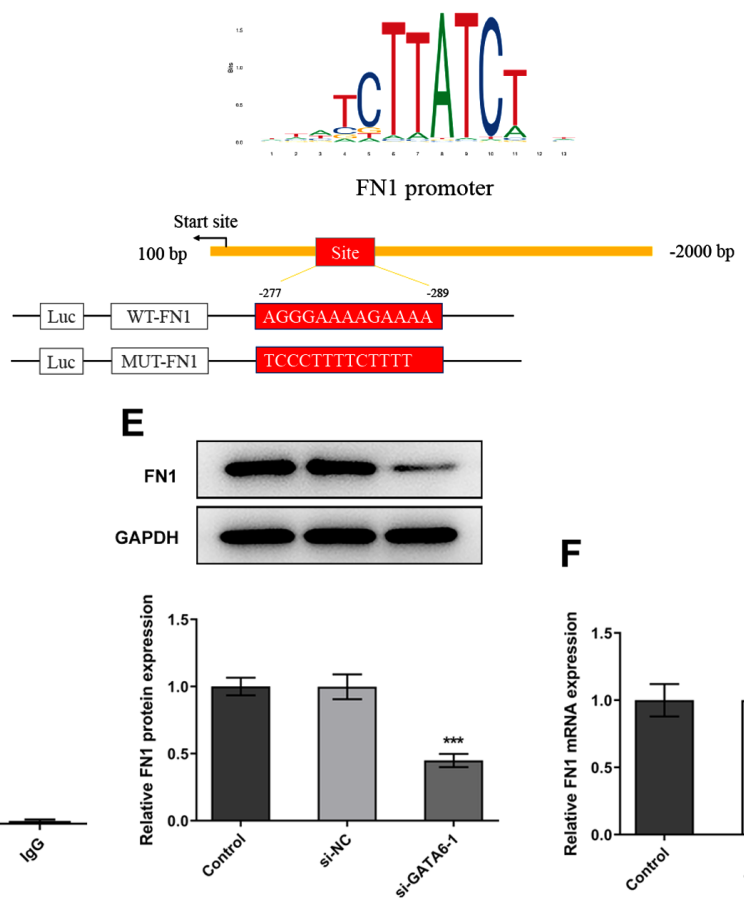

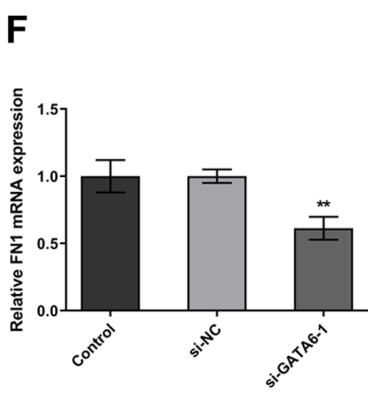

Figure 5. FN1 expression is induced by GATA6. (A) The expression of FN1 in oral squamous cell carcinoma tissues and adjacent tissues was evaluated with TNMplot data. (B) JASPAR database analyses revealed the binding of GATA6 with the FN1 promoter. (C) Binding of GATA6 to the FN1 promoter region was examined using a dual-luciferase reporter assay. ${ }^{* * * *} \mathrm{P}<0.001$ vs. Oe-NC. (D) Direct binding of GATA6 to the FN1 promoter was verified in Cal-27 cells using a chromosomal immunoprecipitation assay. Expression levels of FN1 following GATA6 knockdown were determined using (E) western blot analysis and (F) reverse transcription-quantitative PCR. ${ }^{* *} \mathrm{P}<0.01,{ }^{* * *} \mathrm{P}<0.001$ vs. si-NC. FN1, fibronectin 1; GATA6, GATA binding protein 6; si, small interfering RNA; $\mathrm{Oe}$, overexpression; NC, negative control; Luc, luciferase; wt, wild-type; mut, mutant. 

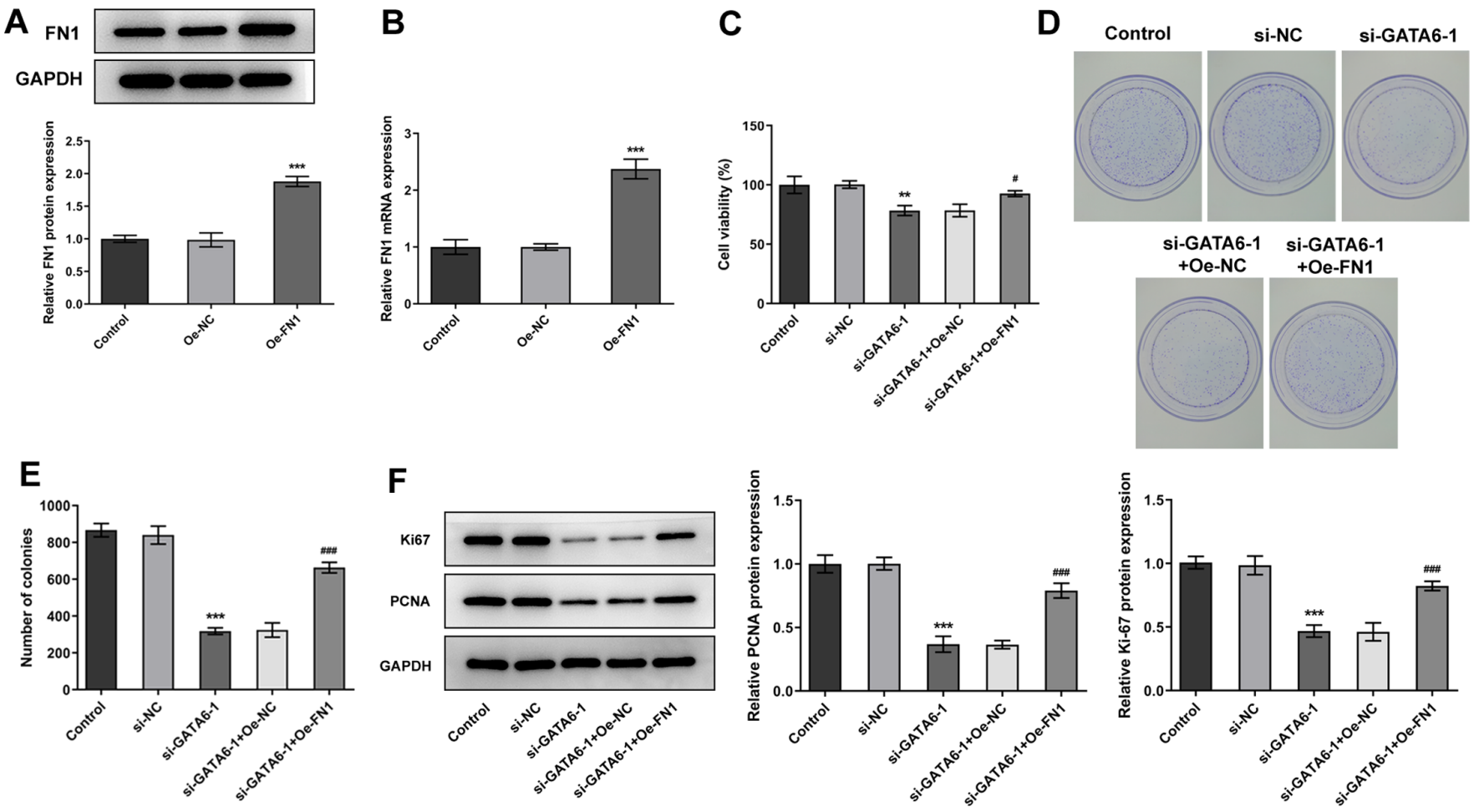

Figure 6. GATA6 silencing suppresses the proliferation and colony formation of oral squamous cell carcinoma cells by regulating FN1. Expression levels of FN1 were detected using (A) western blot analysis and (B) reverse transcription-quantitative PCR following transfection with Oe-FN1. ${ }^{* * *} \mathrm{P}<0.001$ vs. Oe-NC. (C) Cell viability was examined using a Cell Counting Kit-8 assay. (D and E) Cell proliferation was evaluated using a colony formation assay. (F) Western blot analysis was used to assess the expression levels of Ki67 and PCNA. ${ }^{* *} \mathrm{P}<0.01,{ }^{* * * *} \mathrm{P}<0.001$ vs. si-NC; ${ }^{*} \mathrm{P}<0.05$, ${ }^{\# \#} \mathrm{P}<0.001$ vs. si-GATA6-1 + Oe-NC. GATA6, GATA binding protein 6; FN1, fibronectin 1; si, small interfering RNA; Oe, overexpression; NC, negative control; PCNA, proliferating cell nuclear antigen.

by GATA6, thereby inducing tumorigenesis (11). The results of previous studies also demonstrated the involvement of GATA6 in lung cancer (21) and the effectiveness of GATA6 in the reprogramming of hepatocellular carcinoma cells (22). Deletion of GATA6 aided lymphatic metastasis in bladder cancer, thus indicating that GATA6 serves a key role in the lymphatic dissemination of bladder cancer (23). The expression levels of GATA6 vary in a number of types of cancer, for example, expression levels are markedly reduced in liver, ovarian and gastric cancers, compared with notably high expression levels detected in breast cancer and head and neck cancer (24). Additionally, GATA6 expression is markedly elevated in oral carcinoma cell lines (12). The dysregulation of GATA6 in a number of types of cancer is a result of varying GATA6 gene targets (23). Furthermore, regulation of miR-506 expression represses the expression levels of GATA6, thus suppressing the development of OSCC, and overexpression of GATA6 can attenuate the inhibitory effects of miR-506 on cell viability, colony formation, migration and invasion of OSCC cells (13). Additionally, downregulation of GATA6 markedly decreases the expression levels of MMP2 and MMP9 in a murine model of asthma (25). In the present study, GATA6 expression levels were markedly upregulated in OSCC cells. In addition, Cal-27 cells transfected with si-GATA6-1 demonstrated decreased cell proliferation, colony formation, migration and invasion, accompanied by downregulated expression of Ki67, PCNA, MMP2 and MMP9.

In the present study, GSEA revealed that GATA6 was positively associated with FN1. According to the JASPAR database analyses, GATA6 has the ability to bind to the promoter of FN1. FN1, a glycoprotein present in the extracellular matrix, serves a key role in cellular adhesion, migration and tissue remodeling (26). Elevated expression of FN1 was demonstrated in renal and colorectal cancer cells, indicating that FN1 may serve a role in the progression of cancer to an advanced stage $(27,28)$. The results of a previous study further demonstrated the role of FN1 in cell metastasis, differentiation and adhesion in a number of types of cancer (29). In addition, previous studies have labeled FN1 as an oncogene in tumorigenesis and tumor progression and a regulator in physiological processes (30-32). Notably, inhibition of FN1 suppresses the aggressiveness of hepatocellular carcinoma and cervical cancer cells (33). The results of a previous study demonstrate an association between abnormally high expression levels of FN1 and the tumor size and poor prognosis associated with gastric cancer (34). High expression levels of FN1 are also demonstrated in lung tumor growth and survival and resistance to therapy (35). Quantitative proteomics analyses revealed FN1 as a candidate biomarker of sporadic medullary thyroid cancer (36) and FN1 is also considered as a potential biomarker in OSCC regarding the tongue, mouth floor and edentulous ridge (15). Ji et al (37) revealed that the protein expression levels of Ki67 and PCNA is decreased by the silencing of FN1 in human trophoblasts. FN1 knockdown represses cell proliferation, invasion and migration of nasopharyngeal carcinoma cells, coupled with downregulated expression of MMP2 and MMP9 (26). Furthermore, downregulation of FN1 inhibits the development of OSCC (16). Although the role of FN1 in cancer progression has previously been identified, the association between GATA6 and FN1 expression in Cal-27 cells remains 

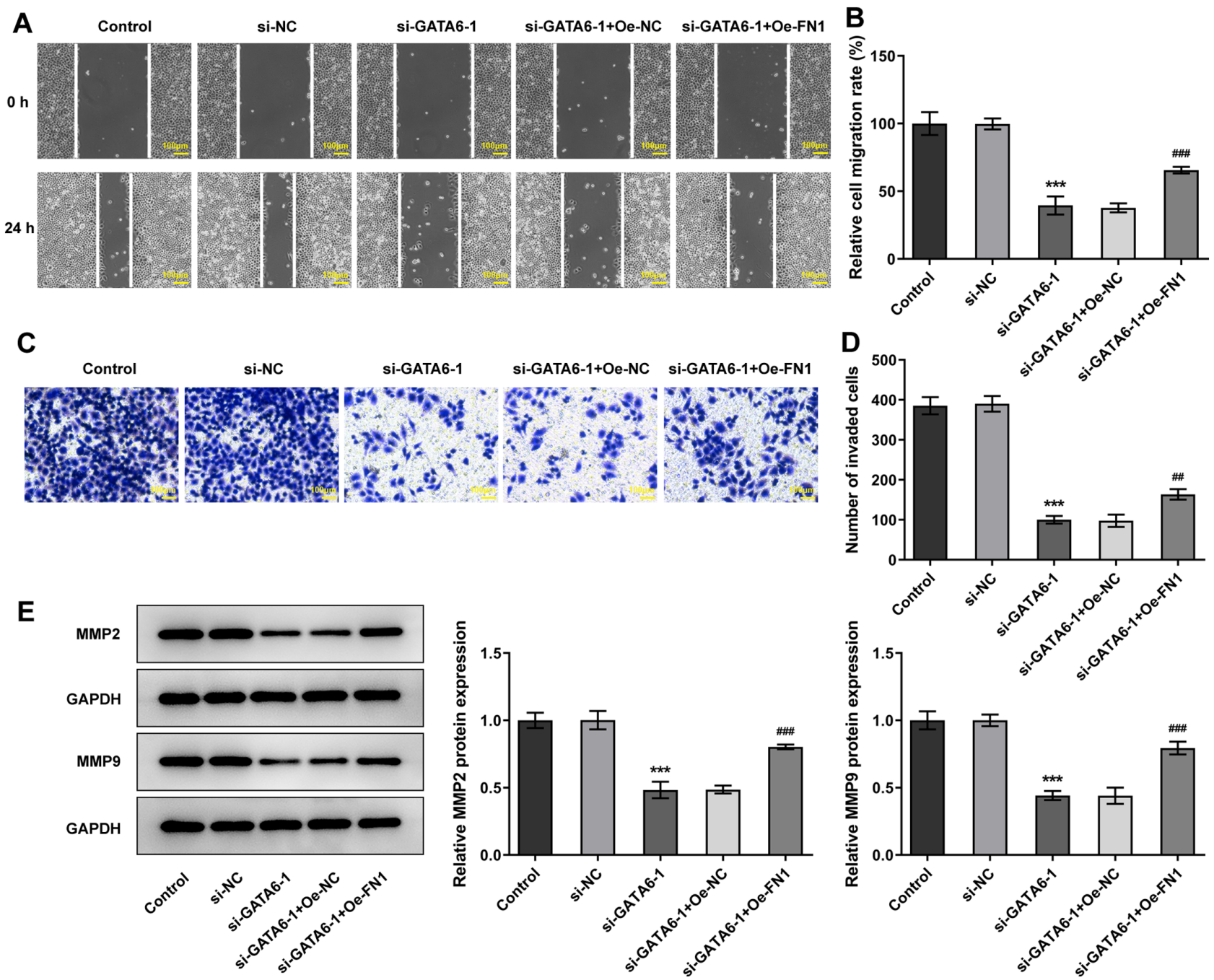

Figure 7. GATA6 silencing suppressed the invasion and migration of oral squamous cell carcinoma cells by regulating FN1. (A and B) Cell migration and $(\mathrm{C}$ and $\mathrm{D})$ invasion were evaluated using wound healing and Transwell assays, respectively. Magnification, $\mathrm{x} 100$. (E) MMP2 and MMP9 expression levels were detected using western blot analysis. ${ }^{* * *} \mathrm{P}<0.001$ vs. si-NC; ${ }^{\# \#} \mathrm{P}<0.01,{ }^{\# \#} \mathrm{P}<0.001$ vs. si-GATA6-1 + Oe-NC. GATA6, GATA binding protein 6; si, small interfering RNA; NC, negative control; FN1, fibronectin 1; Oe, overexpression.

to be elucidated, to the best of the authors' knowledge. Thus, the results of the present study demonstrated that silencing of GATA6 suppressed the proliferation, migration and invasion of Cal-27 cells, which was partly abolished following FN1 overexpression. Therefore, it was hypothesized that silencing GATA6 suppressed the proliferation, migration and invasion of Cal-27 cells by regulating FN1 expression.

In conclusion, the present study is the first, to the best of the authors' knowledge, to demonstrate that GATA6 silencing inhibits the proliferation, migration and invasion of OSCC cells. GATA6 was determined to have a direct regulatory effect on the transcription of FN1 by binding to the FN1 promotor. The results of the present study may contribute to further understanding the pathogenesis of OSCC and provide potential therapeutic targets for the clinical treatment of OSCC. Nevertheless, there are also some limitations to the current study. For instance, whether signaling pathways in the downstream of FN1 are involved in the development of OSCC remains to be elucidated, and whether other potential regulatory mechanisms exist in the regulation of the proliferation, migration and invasion of OSCC cells also needs to be further explored.

\section{Acknowledgements}

Not applicable.

\section{Funding}

No funding was received.

\section{Availability of data and materials}

The datasets used and/or analyzed during the current study are available from the corresponding author on reasonable request.

\section{Authors' contributions}

JZ and GL designed the experimental study. JZ analyzed the experimental data and wrote the manuscript. GL helped to correct the manuscript. JZ carried out the experiments. JZ and GL confirmed the authenticity of all the raw data. Both the authors have read and approved the final manuscript for submission. 


\section{Ethics approval and consent to participate}

Not applicable.

\section{Patient consent for publication}

Not applicable.

\section{Competing interests}

The authors declare that they have no competing interests.

\section{References}

1. Chou $\mathrm{CH}$, Chou YE, Chuang CY, Yang SF and Lin CW: Combined effect of genetic polymorphisms of AURKA and environmental factors on oral cancer development in Taiwan. PLoS One 12: e0171583, 2017.

2. Reyes-Gibby CC, Anderson KO, Merriman KW, Todd KH Shete SS and Hanna EY: Survival patterns in squamous cell carcinoma of the head and neck: Pain as an independent prognostic factor for survival. J Pain 15: 1015-1022, 2014.

3. Lee WH, Chen HM, Yang SF, Liang C, Peng CY, Lin FM, Tsai LL, Wu BC, Hsin CH, Chuang CY, et al: Bacterial alterations in salivary microbiota and their association in oral cancer. Sci Rep 7: 16540, 2017.

4. Dave K, Ali A and Magalhaes M: Increased expression of PD-1 and PD-L1 in oral lesions progressing to oral squamous cell carcinoma: A pilot study. Sci Rep 10: 9705, 2020.

5. Haddad RI and Shin DM: Recent advances in head and neck cancer. N Engl J Med 359: 1143-1154, 2008.

6. Tremblay M, Sanchez-Ferras O and Bouchard M: GATA transcription factors in development and disease. Development 145 dev164384, 2018

7. Jiang X, Li D, Shen W, Shen X and Liu Y: LncRNA NEAT1 promotes hypoxia-induced renal tubular epithelial apoptosis through downregulating miR-27a-3p. J Cell Biochem 120: 16273-16282, 2019.

8. Martinelli P, Carrillo-de Santa Pau E, Cox T, Sainz B Jr, Dusetti N, Greenhalf W, Rinaldi L, Costello E, Ghaneh P, Malats N, et al: GATA6 regulates EMT and tumour dissemination, and is a marker of response to adjuvant chemotherapy in pancreatic cancer. Gut 66: 1665-1676, 2017.

9. Deng X, Jiang P, Chen J, Li J, Li D, He Y, Jiang Y, Zhang Y, Xu S, Li X, et al: GATA6 promotes epithelial-mesenchymal transition and metastasis through MUC1/ $\beta$-catenin pathway in cholangiocarcinoma. Cell Death Dis 11: 860, 2020.

10. Sulahian R, Casey F, Shen J, Qian ZR, Shin H, Ogino S, Weir BA, Vazquez F, Liu XS, Hahn WC, et al: An integrative analysis reveals functional targets of GATA6 transcriptional regulation in gastric cancer. Oncogene 33: 5637-5648, 2014.

11. Tsuji S, Kawasaki Y, Furukawa S, Taniue K, Hayashi T, Okuno M, Hiyoshi M,Kitayama J and Akiyama T: The miR-363-GATA6-Lgr5 pathway is critical for colorectal tumourigenesis. Nat Commun 5: $3150,2014$.

12. Xu CL, Guan WQ and Wang XY: The expression of the GATA6 gene in oral carcinoma cell lines. World J Surg Oncol 19: 153, 2021.

13. Deng L and Liu H: MicroRNA-506 suppresses growth and metastasis of oral squamous cell carcinoma via targeting GATA6. Int J Clin Exp Med 8: 1862-1870, 2015.

14. Gao W, Liu Y, Qin R, Liu D and Feng Q: Silence of fibronectin 1 increases cisplatin sensitivity of non-small cell lung cancer cell line. Biochem Biophys Res Commun 476: 35-41, 2016.

15. Yen CY, Huang CY, Hou MF, Yang YH, Chang CH, Huang HW, Chen $\mathrm{CH}$ and Chang HW: Evaluating the performance of fibronectin 1 (FN1), integrin $\alpha 4 \beta 1$ (ITGA4), syndecan-2 (SDC2), and glycoprotein CD44 as the potential biomarkers of oral squamous cell carcinoma (OSCC). Biomarkers 18: 63-72, 2013.

16. Chen Z, Tao Q, Qiao B and Zhang L: Silencing of LINC01116 suppresses the development of oral squamous cell carcinoma by up-regulating microRNA-136 to inhibit FN1. Cancer Manag Res 11: 6043-6059, 2019.

17. Bartha Á and Győrffy B: TNMplot.com: A web tool for the comparison of gene expression in normal, tumor and metastatic tissues. Int J Mol Sci 22: 2622, 2021
18. Vasaikar SV, Straub P, Wang J and Zhang B: LinkedOmics: Analyzing multi-omics data within and across 32 cancer types. Nucleic Acids Res 46: D956-D963, 2018.

19. Livak KJ and Schmittgen TD: Analysis of relative gene expression data using real-time quantitative PCR and the 2(-Delta Delta C(T)) method. Methods 25: 402-408, 2001.

20. Allison TF, Smith AJH, Anastassiadis K, Sloane-Stanley J, Biga V, Stavish D, Hackland J, Sabri S, Langerman J, Jones M, et al: Identification and single-cell functional characterization of an endodermally biased pluripotent substate in human embryonic stem cells. Stem Cell Reports 10: 1895-1907, 2018

21. Liang G, Meng W, Huang X, Zhu W, Yin C, Wang C, Fassan M, Yu Y, Kudo M, Xiao S, et al: miR-196b-5p-mediated downregulation of TSPAN12 and GATA6 promotes tumor progression in non-small cell lung cancer. Proc Natl Acad Sci USA 117: 4347-4357, 2020.

22. Tan HW, Leung CO, Chan KK, Ho DW, Leung MS, Wong CM, $\mathrm{Ng}$ IO and Lo RC: Deregulated GATA6 modulates stem cell-like properties and metabolic phenotype in hepatocellular carcinoma. Int J Cancer 145: 1860-1873, 2019.

23. Wang C, Liu Q, Huang M, Zhou Q, Zhang X, Zhang J, Xie R, Yu Y, Chen S, Fan J, et al: Loss of GATA6 expression promotes lymphatic metastasis in bladder cancer. FASEB J 34: 5754-5766, 2020.

24. Cheung WK, Zhao M, Liu Z, Stevens LE, Cao PD, Fang JE, Westbrook TF and Nguyen DX: Control of alveolar differentiation by the lineage transcription factors GATA6 and HOPX inhibits lung adenocarcinoma metastasis. Cancer Cell 23: 725-738, 2013.

25. Fang P, Shi HY, Wu XM, Zhang YH, Zhong YJ, Deng WJ, Zhang YP and Xie M: Targeted inhibition of GATA-6 attenuates airway inflammation and remodeling by regulating caveolin-1 through TLR2/MyD88/NF- $\mathrm{BB}$ in murine model of asthma. Mol Immunol 75: 144-150, 2016.

26. Ding Y, Pan Y, Liu S, Jiang F and Jiao J: Elevation of MiR-9-3p suppresses the epithelial-mesenchymal transition of nasopharyngeal carcinoma cells via down-regulating FN1, ITGB1 and ITGAV. Cancer Biol Ther 18: 414-424, 2017.

27. Steffens S, Schrader AJ, Vetter G, Eggers H, Blasig H, Becker J, Kuczyk MA and Serth J: Fibronectin 1 protein expression in clear cell renal cell carcinoma. Oncol Lett 3: 787-790, 2012

28. Wu J, Wang Y, Xu X, Cao H, Sahengbieke S, Sheng H, Huang Q and Lai M: Transcriptional activation of FN1 and IL11 by HMGA2 promotes the malignant behavior of colorectal cancer. Carcinogenesis 37: 511-521, 2016.

29. Shi H, Dong Z and Gao H: LncRNA TUG1 protects against cardiomyocyte ischaemia reperfusion injury by inhibiting HMGB1. Artif Cells Nanomed Biotechnol 47: 3511-3516, 2019.

30. Cai X, Liu C, Zhang TN, Zhu YW, Dong X and Xue P: Down-regulation of FN1 inhibits colorectal carcinogenesis by suppressing proliferation, migration, and invasion. J Cell Biochem 119: 4717-4728, 2018.

31. Xie Y, Liu C, Qin Y, Chen J and Fang J: Knockdown of IRE1a suppresses metastatic potential of colon cancer cells through inhibiting FN1-Src/FAK-GTPases signaling. Int J Biochem Cell Biol 114: 105572, 2019

32. Song G, Liu K, Yang X, Mu B, Yang J, He L, Hu X, Li Q, Zhao Y, Cai X, et al: SATB1 plays an oncogenic role in esophageal cancer by up-regulation of FN1 and PDGFRB. Oncotarget 8: 17771-17784, 2017.

33. Xu X, Liu Z, Zhou L, Xie H, Cheng J, Ling Q, Wang J, Guo H, Wei X and Zheng S: Characterization of genome-wide TFCP2 targets in hepatocellular carcinoma: Implication of targets FN1 and TJP1 in metastasis. J Exp Clin Cancer Res 34: 6, 2015.

34. Sun Y, Zhao C, Ye Y, Wang Z, He Y, Li Y and Mao H: High expression of fibronectin 1 indicates poor prognosis in gastric cancer. Oncol Lett 19: 93-102, 2020.

35. Han S, Khuri FR and Roman J: Fibronectin stimulates non-small cell lung carcinoma cell growth through activation of Akt/mammalian target of rapamycin/S6 kinase and inactivation of LKB1/AMP-activated protein kinase signal pathways. Cancer Res 66: 315-323, 2006.

36. Zhan S, Li J, Wang T and Ge W: Quantitative proteomics analysis of sporadic medullary thyroid cancer reveals FN1 as a potential novel candidate prognostic biomarker. Oncologist 23: 1415-1425, 2018

37. Ji J, Chen L, Zhuang Y, Han Y, Tang W and Xia F: Fibronectin 1 inhibits the apoptosis of human trophoblasts by activating the PI3K/ Akt signaling pathway. Int J Mol Med 46: 1908-1922, 2020

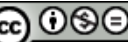

This work is licensed under a Creative Commons Attribution-NonCommercial-NoDerivatives 4.0 International (CC BY-NC-ND 4.0) License. 